LA GRANJA:

REVISTA DE

CIENCIAS DE LA VIDA

pISSN:1390-3799; eISSN:1390-8596

http:/ / doi.org/10.17163/lgr.n32.2020.06
Artículo científico / Scientific paper

MANEJO DE DESECHOS

\title{
SUSTENTABILIDAD Y EVALUACIÓN DEL IMPACTO OCASIONADO POR EL RELLENO SANITARIO DEL MUNICIPIO DE CARMEN EN CAMPECHE, MÉXICO
}

\author{
SUSTAINABILITY AND EVALUATION OF THE IMPACT CAUSED BY \\ THE LANDFILL OF THE MUNICIPALITY OF CARMEN, CAMPECHE, \\ MÉXICO
}

\author{
Areli Machorro-Román ${ }^{1}{ }^{\oplus}$, Genoveva Rosano-Ortega ${ }^{1}{ }^{\oplus}$, María Elena \\ Tavera-Cortés $^{2}{ }^{\circledR}$, Juan Gabriel Flores-Trujillo ${ }^{3}$, María Rosa \\ Maimone-Celorio $^{1}{ }^{\oplus}$, Estefanía Martínez-Tavera ${ }^{1}{ }^{1}$, Sonia

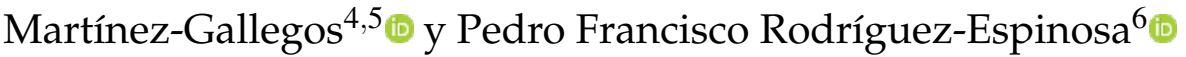

\footnotetext{
${ }^{1}$ Facultad de Ingeniería Ambiental, Universidad Popular Autónoma del Estado de Puebla. Av. 21 sur, 1103, Barrio de Santiago, 72410, Puebla, México.

2 Instituto Politécnico Nacional, Av. Luis Enrique Erro S/N, Unidad Profesional Adolfo López Mateos, Zacatenco, Alcaldía Gustavo A. Madero, 07738, Ciudad de México, México.

${ }^{3}$ Facultad de Ingeniería, Universidad Autónoma del Carmen. Av. 56 No. 4 Esq. Avenida Concordia Col. Benito Juárez, 24180, Ciudad del Carmen, Campeche, México.

${ }^{4}$ Tecnológico Nacional de México, Avenida Universidad 1200, Colonia Xoco, Coyoacán, 03330, Ciudad de México, México.

${ }^{5}$ Instituto Tecnólogico de Toluca, Av. Tecnológico s/n. Colonia Agrícola Bellavista Metepec, 52149, Edo. De México, México.

${ }^{6}$ Centro Interdisciplinario de Investigaciones y Estudios sobre Medio Ambiente y Desarrollo (IPN-CIIEMAD), Instituto Politécnico Nacional. Calle 30 dejunio de 1520, Barrio de la Laguna Ticomán, Del. Gustavo A Madero, C.P. 07340, Ciudad de México, México.

*Autor para correspondencia: mtavera@ipn.mx
}

\begin{abstract}
Resumen
Los indicadores de sustentabilidad permiten evaluar los impactos ambientales relacionados con la estrategia del desarrollo sustentable. Este estudio se realizó en Ciudad del Carmen, Campeche, que es considerada una isla de barrera que se localiza al sureste de México. El municipio canaliza la disposición final de los residuos sólidos urbanos (RSU) a través de un relleno sanitario el cual se encuentra ubicado en una zona de manglar, teniendo un impacto negativo en el medio ambiente lo que incide negativamente en el desarrollo sustentable. Mediante el análisis sedimentológico del subsuelo, se obtuvieron resultados que identificaron la dominancia de arenas carbonatadas, lo que permitió definir un rango de porosidad del 20,2 al 40,1\%, y permeabilidad de $\pm 10^{-2}-10^{-4} \mathrm{~ms}^{-1}$ darcys, es decir, los sedimentos
\end{abstract}


presentan una buena porosidad y una permeabilidad alta. Por su parte, mediante un análisis de la calidad del agua se detectaron concentraciones de Demanda Bioquímica de Oxígeno $\left(D B O_{5}\right)$ y Demanda Química de Oxígeno (DQO) de 63,06 y 1338,13 $\mathrm{mg} \mathrm{L}^{-1}$, respectivamente, así como la presencia de concentraciones de trazas de algunos metales pesados. Estos valores permitieron clasificarlo como un cuerpo de agua fuertemente contaminado.

Palabras clave: México, sustentabilidad, residuos, lixiviados, contaminación, agua, sedimentos

\begin{abstract}
The sustainability indicators allow the evaluation of the environmental impacts related to the sustainable development strategy. The research was conducted in ciudad del Carmen, Campeche, whichis considered a barrier island that is located at the southeast of Mexico. The municipality channels the final disposal of solid urban waste (MSW) through a sanitary landfill which is located in a mangrove area, having a negative impact on the environment, which negatively affects the sustainable development. This research identified a sequence of carbonated sands by means of subsoil sediment analysis, which allowed to define a porosity of 20.2 to $40.1 \%$ and a permeability of $\pm 10^{-2}-10^{-4}$ $\mathrm{ms}^{-1}$, i.e., the sediments have good porous and high permeability. On the other hand, and with respect to water quality, concentrations of $B O D_{5}$ and COD in the mangrove were 63.06 and $1338.13 \mathrm{mg} \mathrm{L}^{-1}$, respectively, as well as the presence of trace concentrations of some heavy metals. These values allowed to classify it as a strongly contaminated body of water.
\end{abstract}

Keywords: Mexico, sustainability, waste, leachate, pollution, water, sediments

\footnotetext{
Forma sugerida de citar: Machorro, A., Rosano, G., Tavera, M., Flores, J., Maimone, M., Martínez, E., Martínez, S. y Rodríguez, P. (2020). Sustentabilidad y evaluación del impacto ocasionado por el relleno sanitario del Municipio del Carmen en campeche, México. La Granja: Revista de Ciencias de la Vida. Vol. 32(2):72-92. http:/ / doi.org/10.17163/lgr.n32. 2020.06 .
}

IDs Orcid:

Areli Machorro Román: http:/ / orcid.org/0000-0003-4666-4489

Genoveva Rosano-Ortega: http:/ / orcid.org/0000-0002-7297-3456

María Elena Tavera-Cortés: http:/ / orcid.org/0000-0002-2179-2735

Juan Gabriel Flores-Trujillo: http:/ / orcid.org/0000-0002-6221-216X

María Rosa Maimone Celorio: http:/ / orcid.org/0000-0002-9638-1578

Sonia Martínez-Gallegos: http:/ / orcid.org/0000-0002-7297-3456

Pedro Francisco Rodríguez Espinosa: http:/ / orcid.org/0000-0002-0443-5728

Estefanía Martínez Tavera: http:/ /orcid.org/0000-0003-0449-037X 


\section{Introducción}

Ciudad del Carmen, Campeche, es una isla de barrera de origen sedimentario de edad Cuaternaria (2,58 Ma. aprox.) (SGM, 2005; CONABIO, 2012; ICS, 2018) localizada al sureste de México (Figura 1). La isla forma parte del sistema de lagunas estuarinas más grande y valiosa de México: la Laguna de Términos (Escudero, Silva y Mendoza, 2014). Este siste- ma presenta una forma elipsoide con una longitud de $70 \mathrm{Km}$ y un ancho de $30 \mathrm{Km}$, y cubre un área de $2500 \mathrm{Km}^{2}$, con una profundidad promedio de 3-3,5 $\mathrm{m}$. Los principales ríos que depositan sedimentos en este sistema lagunar son el río Palizada $\left(240 \mathrm{~m}^{3}\right.$ $\left.\mathrm{s}^{-1}\right)$, el río Candelaria $\left(35 \mathrm{~m}^{3} \mathrm{~s}^{-1}\right)$ y el río Chumpán $\left(2 \mathrm{~m}^{3} \mathrm{~s}^{-1}\right)$, el cual provee una descarga significativa de agua fresca de $400 \mathrm{~m}^{3} \mathrm{~s}^{-1}$ (Magallanes-Ordóñez y col., 2015).

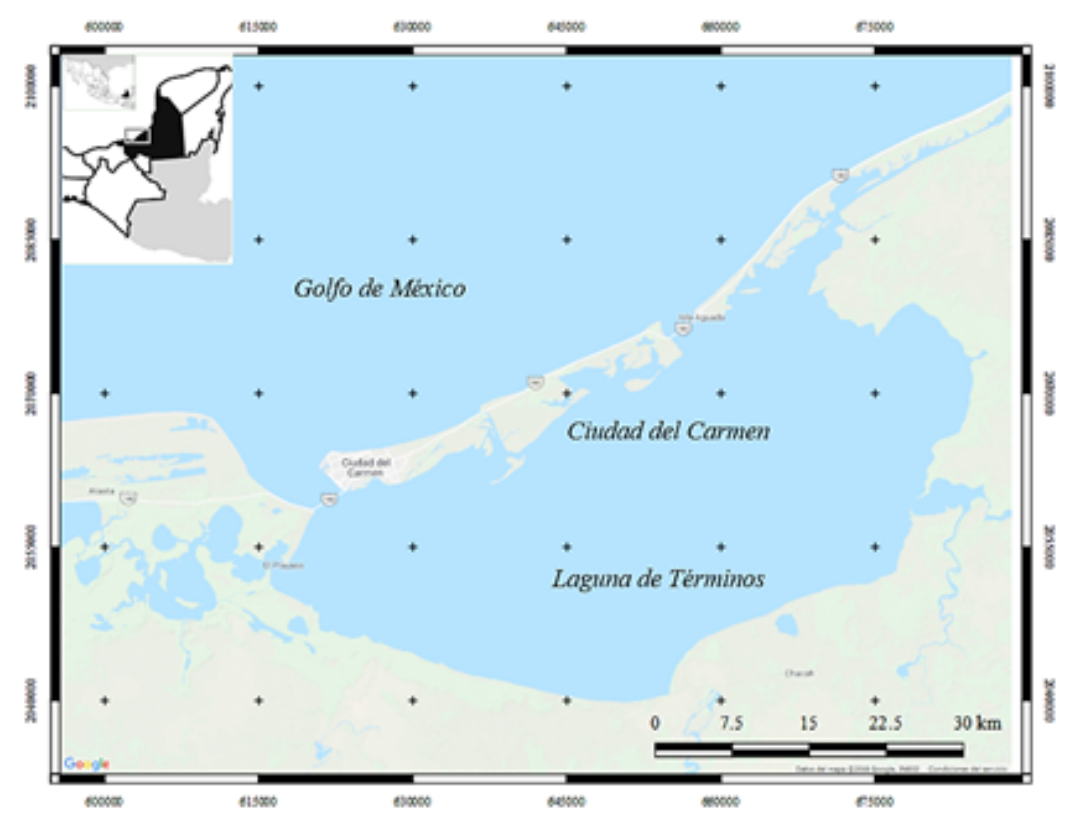

Figura 1. Ubicación de Ciudad del Carmen, Campeche (Modificado de INEGI (2010).)

La isla posee gran importancia ecológica debido a que está dominada por un ecosistema de tipo manglar (manglar "Isla del Carmen"; 43,92 $\mathrm{Km}^{2}$ ) que se extiende a lo largo de 43,92 $\mathrm{Km}^{2}$ (4392 ha) (CONABIO, 2012) y forma parte del sitio RAMSAR denominado "Área de Protección de Flora y Fauna Laguna de Términos", el cual posee una superficie de 705,016 ha que incluye a la isla, la Laguna de Términos y algunas porciones que las rodean $(\mathrm{CO}-$ NANP, 2018).

Los humedales representan uno de los tipos de ecosistemas más importantes del mundo, y son también uno de los más amenazados ( $\mathrm{Hu}$ y col., 2017). Este tipo de ecosistemas son ecológicamente importantes porque fungen como hábitat para diversas comunidades de peces y vida silvestre, así como de diversas especies comercialmente valiosas, y son amortiguadores de la línea de costa, proveen agua dulce, reducen la sedimentación en aguas navegables, y ayudan potencialmente en el almacenamiento de las aguas de inundación. Aunado a esto, sus suelos contienen algunas de las mayores reservas de carbono en la biósfera (Maynard, Dahlgren y O'Geen, 2014; Ghosh, Mishra y Gitelson, 2016; Nahlik y Fennessy, 2016; Rains y col., 2016).

\subsection{Litología}

El área de estudio se localiza a $8 \mathrm{Km}$ (aprox.) de Ciudad del Carmen, en las inmediaciones del relleno sanitario municipal (Figura 1), antiguo delta interno Cocoyotes-el Cayo (Palacio-Prieto, OrtízPérez y Garrido-Pérez, 1999). Este relleno sanitario 
está rodeado por una serie de canales que desembocan en la Laguna de Términos (dirección de corriente N-S), todos estos canales fueron parte del antiguo delta interno.

La isla de Carmen (de edad Cuaternaria) está formada por una planicie costera al norte, conformada por cordones de playa altos y bajos sobre sedimentos arenosos, y una planicie baja de inundación lagunar al sur dominada por una llanura fluvio-marina sobre sedimentos arcillo limosos (Ramos-Reyes y col., 2016). Litológicamente la isla está constituida por una secuencia de sedimentos de origen litoral al norte, y una secuencia de sedimentos de origen palustre al sur; en la Laguna de Términos se reportan limos ricos en materia orgánica, así como arenas ricas en aluminosilicatos y carbonatos derivados del suministro aluvial del mar y de la isla de Carmen (Darnell, 2015; Jones, 2015; Magallanes-Ordóñez y col., 2015).

Los depósitos palustres se caracterizan por un nivel de energía bajo. En estos ambientes la dinámica de los sedimentos y todos los procesos están vinculados al régimen de las mareas y las corrientes, que juegan el rol fundamental en este tipo de ambientes, y los limos son predominantes. Por su parte, los depósitos litorales están caracterizados por un nivel elevado de energía, lo cual permite el depósito de arenas (Martínez y col., 2015).

La distribución espacial de los sedimentos es de la siguiente forma: 1) arenas en la costa interior de la isla, 2) arenas limosas en la entrada de la isla (oeste) y en las desembocaduras de los ríos Palizada, Chumpán y Candelaria, y 3) las arenas están presentes en la costa de la isla (Magallanes-Ordóñez y col., 2015).

\subsection{Normatividad}

La normativa general aplicable para la disposición final de residuos corresponde a la NOM-083SEMARNAT-2003 (SEMARNAT, 2003), en el apartado de "Especificaciones para la selección del sitio" y "Restricciones para la ubicación del sitio" menciona que se deben considerar como primordiales tres puntos:

- "No se deben ubicar sitios de disposición final dentro de áreas naturales protegidas. En todo caso deberá observarse lo ordenado en la Declaratoria de creación correspondiente y, en su caso, en la subzonificación y reglas administrativas contenidas en el Programa de Manejo respectivo." Según la CONANP (2018), el relleno sanitario municipal se localiza dentro del Área de Protección de Flora y Fauna Laguna de Términos.

- "No debe ubicarse en zonas de: marismas, manglares, esteros, pantanos, humedales, estuarios, planicies aluviales, fluviales, recarga de acuíferos; ni sobre cavernas, fracturas o fallas geológicas activas." Según la CONABIO (2012), la isla está dominada por un ecosistema de tipo manglar.

- Por otro lado, según el Manual de especificaciones técnicas para la construcción de rellenos sanitarios para RSU y RME (SEMARNAT, 2009), un relleno sanitario debe contar con las siguientes especificaciones técnicas: i) celdas, ii) sistema de impermeabilización, iii) sistema de extracción, captación y control de biogás, y iv) sistema de extracción, captación y control de lixiviados. El relleno sanitario municipal incumple en la ingeniería establecida por este manual, por lo cual su operación compromete la protección ambiental del área.

El objetivo del presente artículo es evaluar el impacto ambiental que genera el relleno sanitario del municipio de Carmen, Camp. en el subsuelo de la región norte central del "Área de Protección de Flora y Fauna Laguna de Términos (APFFLT)" a través de un análisis sedimentológico, para determinar la capacidad de infiltración de los lixiviados en el desarrollo sustentable. Con la finalidad de diferenciar las muestras de sedimento de las de agua, se empleó la clave PV (perfil vertical) para sedimentos y MA (muestra de agua) para agua, respectivamente. 


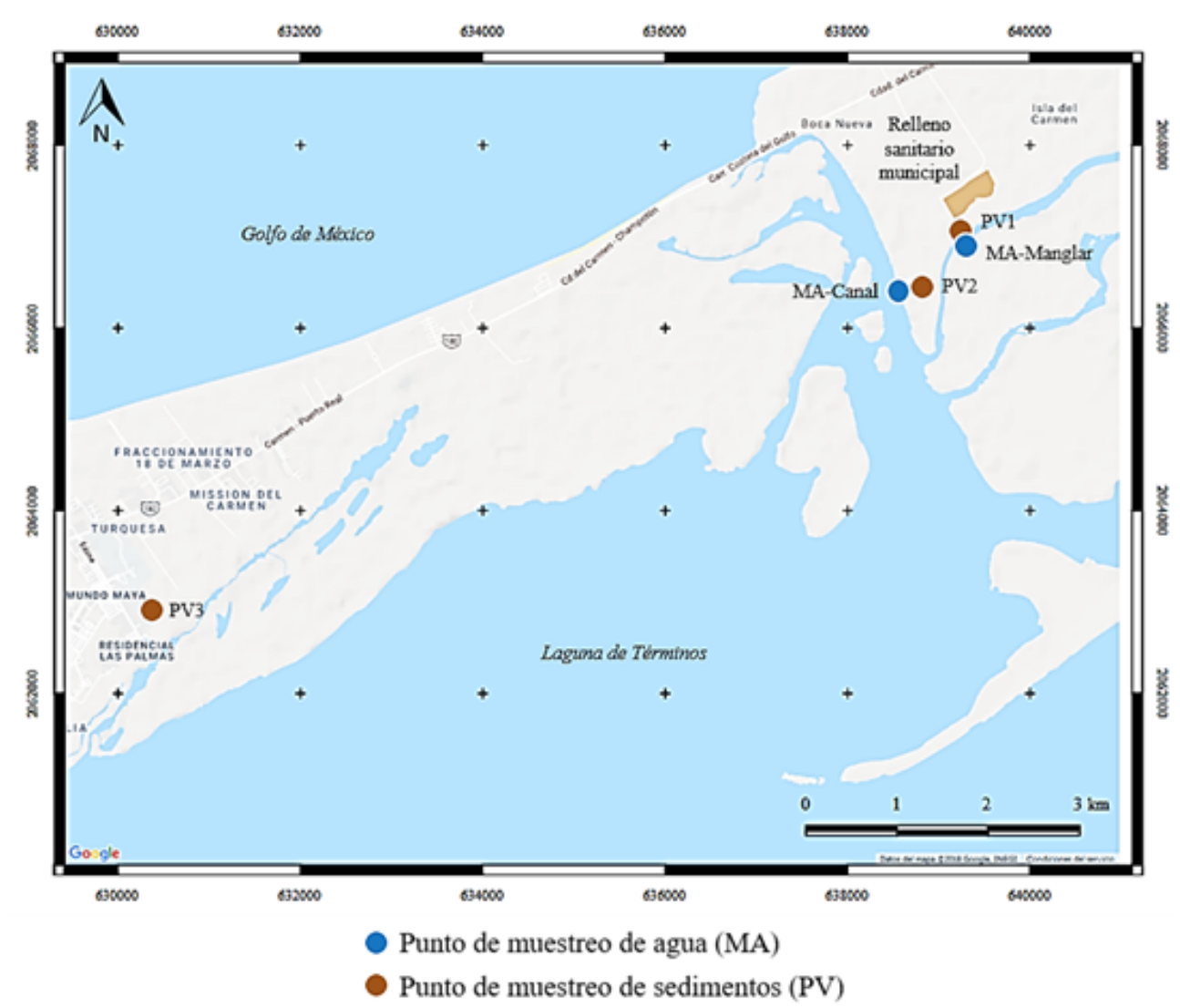

Figura 2. Ubicación de los puntos de muestreo.

\section{Materiales y métodos}

Los indicadores de sustentabilidad (IS) permiten relacionar la información ambiental con la económica y social, a fin de generar información sobre la contaminación, el deterioro del desarrollo productivo o el bienestar alcanzado por la población (Ibáñez-Forés, Bovea y Azapagic, 2013). Conforme a la información del Censo Nacional de Gobiernos Municipales y Delegacionales del Instituto nacional de Estadística y Geografía (INEGI, 2017), en Campeche se recolectan diariamente $792190 \mathrm{Kg}$ de residuos sólidos urbanos (RSU), de los cuales el municipio que mayormente recolecta es Ciudad del Carmen con $289140 \mathrm{Kg}$ diarios lo que representa un total del $36,5 \%$ del total de los residuos recolectados en Campeche; a los RSU no se les aplica ningún tipo de tratamiento ni selección y tienen como destino el relleno sanitario seleccionado en esta investigación.
El muestreo se realizó en época de estiaje, considerando que durante este período los lixiviados presentan mayor movilidad debido al incremento de los escurrimientos producto de las precipitaciones, lo cual se considera factible para el muestreo de la calidad del agua. Las muestras de sedimento se colectaron en 3 estaciones en la periferia del relleno sanitario municipal y dentro de la zona de manglar del área de estudio, tal y como se muestra en la Figura 2, siguiendo la NOM-021-SEMARNAT-2000 que "establece las especificaciones de fertilidad, salinidad y clasificación de suelos, estudios, muestreo y análisis" (SEMARNAT, 2000). Los puntos de colecta fueron seleccionados de acuerdo a la cercanía con el relleno sanitario del municipio de Carmen y aleatoriamente alrededor de este, considerando la uniformidad de las facies sedimentarias a lo largo de la isla. El PV1 se localiza a $\pm 407,25 \mathrm{~m}$ del relleno sanitario $\left(18^{\circ} 41^{\prime} 25,332^{\prime \prime} \mathrm{N}, 9140^{\prime} 46,307^{\prime \prime} \mathrm{O}\right)$. El PV2 se localiza a $\pm 1184,21 \mathrm{~m}$ del relleno sanitario $\left(18^{\circ} 41^{\prime} 5,46^{\prime \prime} \mathrm{N}, 91^{\circ} 41^{\prime} 4,199^{\prime \prime} \mathrm{O}\right)$. Y finalmente el 
PV3 se localiza a $\pm 10104,23 \mathrm{~m}$ del relleno sanitario $\left(18^{\circ} 39^{\prime} 2^{\prime \prime} \mathrm{N}, 91^{\circ} 45^{\prime} 51^{\prime \prime} \mathrm{O}\right)$. El análisis sedimentológico fue realizado según la metodología propuesta por Arellano (2003) y Honarpour (2018). Como primer paso, las muestras fueron sometidas al método de tamizado para el análisis granulométrico, empleando 14 tamices de aberturas -2 a $4 \varphi(4$ a 0,063 $\mathrm{mm})$, cada $0,5 \varphi$. Este procedimiento se realizó en el Laboratorio de Geofísica de la Universidad Autónoma del Carmen (UNACAR). Posteriormente, las muestras fueron trasladadas al Laboratorio de Investigación de Biotecnoambiental de la Universidad Popular Autónoma del Estado de Puebla (UPAEP), en donde se llevó a cabo la determinación de com- ponentes sedimentarios y la determinación de propiedades de masa (porosidad). Tanto las mediciones del tamaño de la partícula como la determinación de porosidad se realizaron por triplicado para la obtención de un dato válido. Mediante la aplicación de estadística descriptiva (Rendón-Macías, VillasísKeeve y Miranda-Novales, 2016), se generaron las correspondientes tablas y gráficas. Dentro del análisis de las condiciones topográficas del área de estudio se implementó un cálculo de trigonometría (por diferenciación de elevaciones) para la obtención del ángulo de inclinación de la zona, y con esto determinar la pendiente de escurrimiento de los lixiviados hasta los canales principales.
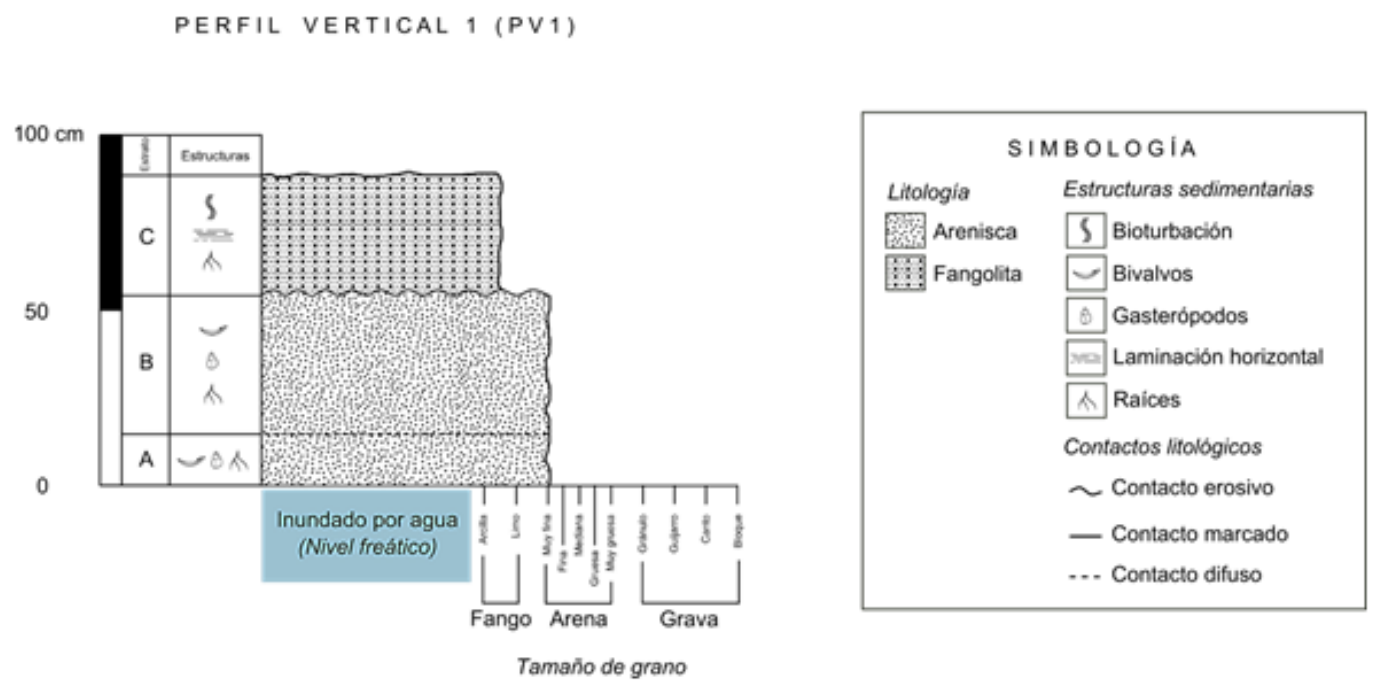

Figura 3. Columna litológica del PV1.

Por su parte, las muestras de agua se colectaron en dos estaciones (Figura 2), según las NMXAA-003-1980 ("Aguas residuales -Muestreo") (SCFI, 1980a) y NMX-AA-014-1980 ("Cuerpos receptores -Muestreo") (SCFI, 1980b), y se preservaron según la NMX-AA-028-SCFI-2001 ("Análisis de agua -Determinación de la Demanda Bioquímica de Oxígeno en aguas naturales, residuales (DBO5) y residuales tratadas -Método de prueba") (SCFI, 2001a) para la DBO5, NMX-AA-030/2-SCFI-2011 ("Análisis de agua -Determinación de la Demanda Química de Oxígeno en aguas naturales, residuales y residuales tratadas -Método de prueba -Parte 2 determinación del índice de la Demanda Química de Oxígeno -Método de tubo sellado a pequeña escala") (SCFI, 2011b) para DQO, NMX-AA-029SCFI-2001 ("Análisis de aguas -Determinación de Fósforo total en aguas naturales, residuales y residuales tratadas - Método de prueba") (SCFI, 2001b) para Fósforo total, NMX-AA-026-SCFI-2010 ("Análisis de agua - Medición de Nitrógeno total Kjeldahl en aguas naturales, residuales y residuales tratadas -Método de prueba") (SCFI, 2011a) para Nitrógeno total Kjeldahl, y NMX-AA-051-SCFI-2016 ("Análisis de agua -Medición de metales por absorción atómica en aguas naturales, potables, residuales y residuales tratadas -Método de prueba") (SCFI, 2016) para metales. Los puntos de colecta fueron seleccionados, el primero, cercano al relleno sanitario del municipio de Carmen $( \pm 481,65 \mathrm{~m}$ del relleno sani- 
tario; coordenadas $18^{\circ} 41^{\prime} 22,452^{\prime \prime} \mathrm{N}, 91^{\circ} 40^{\prime} 45,732^{\prime \prime}$ $\mathrm{O})$, y el segundo considerando el transporte del efluente a través del canal principal (a $\pm 1270,46 \mathrm{~m}$ del relleno sanitario; coordenadas $18^{\circ} 41^{\prime} 4,416^{\prime \prime} \mathrm{N}$, $\left.91^{\circ} 41^{\prime} 7,62^{\prime \prime} \mathrm{O}\right)$.

Es preciso destacar que la relación entre la distancia de los puntos con el relleno sanitario municipal y la topografía de la zona (pendiente a favor del escurrimiento hacia el manglar) permitió evaluar el impacto ambiental. Para la determinación de los parámetros de DBO5, DQO, Fósforo Total y Nitrógeno Total Kjeldahl, las muestras fueron analizadas por Litoral Laboratorios Industriales (Cd. del Carmen, Camp.; con la acreditación EMA: AG-0135-015/11), bajo el cumplimiento de la NOM-001-SEMARNAT1996 que "establece los límites máximos permisibles de contaminantes en las descargas de aguas residuales en aguas y bienes nacionales. Secretaría de Medio Ambiente y Recursos Naturales" (SEMARNAT, 1996). Por su parte, para la determinación de metales pesados, las muestras fueron trasladadas para su análisis en el Laboratorio de Bioingenierías de la UPAEP, en donde se llevó a cabo el análisis cuantitativo realizado a partir de la metodología de espectrofotometría por absorción atómica, según la NMX-AA-051-SCFI-2001 (SCFI, 2001b). Para el análisis de los datos anteriores se aplicó igualmente estadística descriptiva (Rendón-Macías, VillasísKeeve y Miranda-Novales, 2016).

\section{Resultados}

\subsection{Análisis sedimentológico}

\subsubsection{PV1 (Perfil vertical 1)}

Se identificó una columna litológica con un espesor de 0,90 m.s.n.m. conformada por 3 estratos. Granulométricamente, dominan arenas de 0,125 a 0,062 mm compuestas por biógenos calcáreos y materia orgánica. Se determinó una porosidad con un intervalo de 20,2 a 30,2\% (Figura 3).

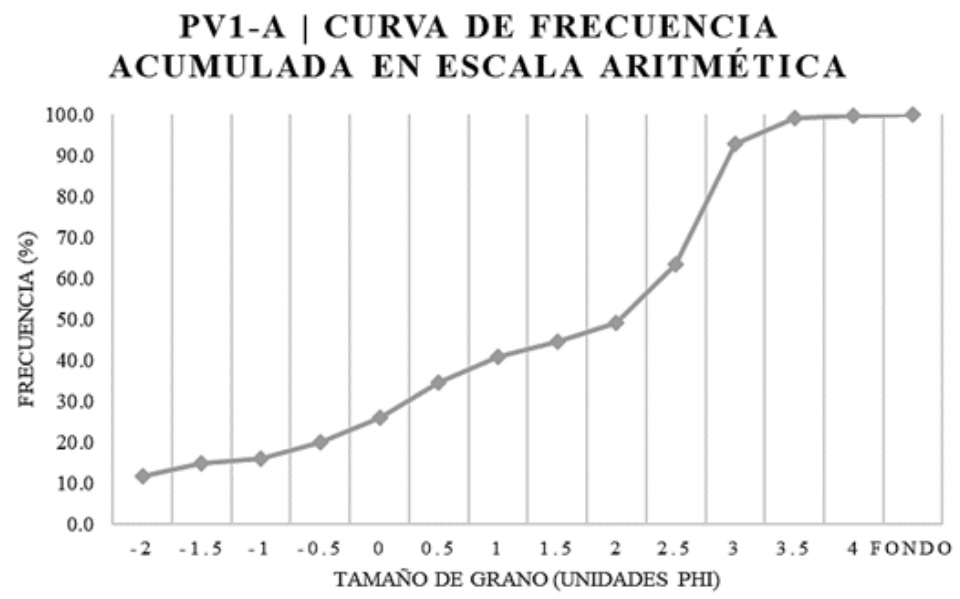

Figura 4. Curva de frecuencia acumulada en escala aritmética del PV1-A.

Estrato A (PV1-A): Dominado por partículas de tamaño de arenas muy finas $(3,5 \varphi$ a $0,125 \mathrm{~mm})$ (Figura 4). Perteneciente al grupo textural Gravelly Sand, clasificado con el nombre del sedimento Fine Gravelly Fine Sand. Composicionalmente dominado por biógenos calcáreos y materia orgánica y con un porcentaje de porosidad de $20,2 \%$.
Estrato B (PV1-B): Dominado por partículas tamaño arenas finas $(2,5 \varphi$ a $0,25 \mathrm{~mm})$ (Figura 5). Perteneciente al grupo textural Gravelly Sand, clasificado con el nombre del sedimento Very Fine Gravelly Fine Sand. Composicionalmente dominado por materia orgánica y cuarzo. Presenta un porcentaje de porosidad de $30,2 \%$. 


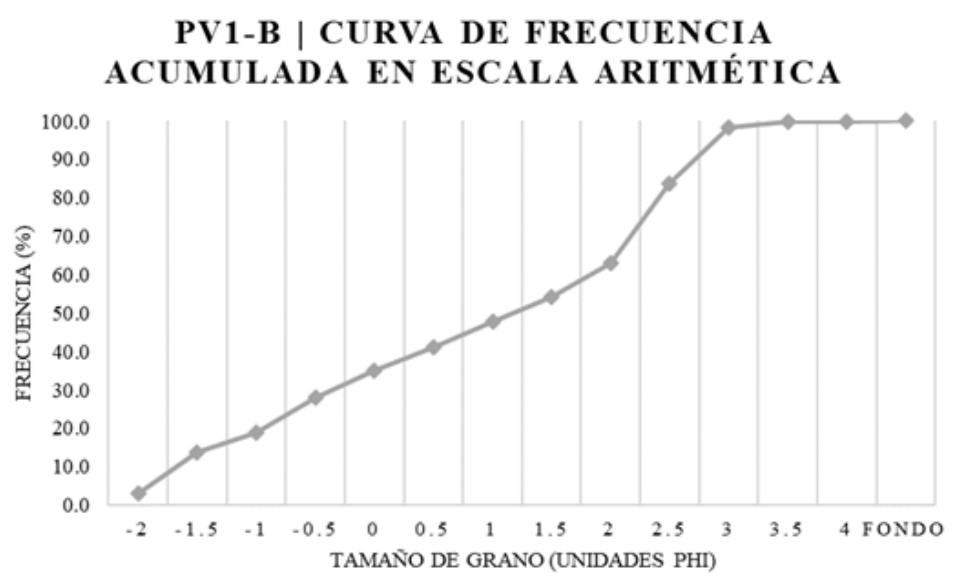

Figura 5. Curva de frecuencia acumulada en escala aritmética del PV1-B.

\subsubsection{PV2 (Perfil vertical 2)}

Se identificó una columna litológica con un espesor de 0,83 m.s.n.m, conformada por 5 estratos. Granulométricamente, dominan arenas de 1 a $0,125 \mathrm{~mm}$ compuestas por biógenos calcáreos y material detrítico. Se determinó una porosidad con un intervalo de 20,3 a $36,5 \%$ (Figura 6 ).

Estrato A (PV2-A): Dominado por partículas tamaño arenas gruesas $(0,5 \varphi$ a $1 \mathrm{~mm})$ (Figura 7$)$. Perteneciente al grupo textural Gravelly Sand, cla- sificado con el nombre del sedimento Fine Gravelly Coarse Sand. Composicionalmente dominado por biógenos calcáreos y cuarzo. Presenta un porcentaje de porosidad de $20,3 \%$.

Estrato B (PV2-B): Dominado por partículas tamaño arenas muy finas a finas $(3,5 \varphi$ a $0,25 \mathrm{~mm})$ (Figura 8). Perteneciente al grupo textural Gravelly Sand, clasificado con el nombre del sedimento Fine Gravelly Fine Sand. Composicionalmente dominado por biógenos calcáreos y fragmentos de roca. Presenta un porcentaje de porosidad de $36,5 \%$.
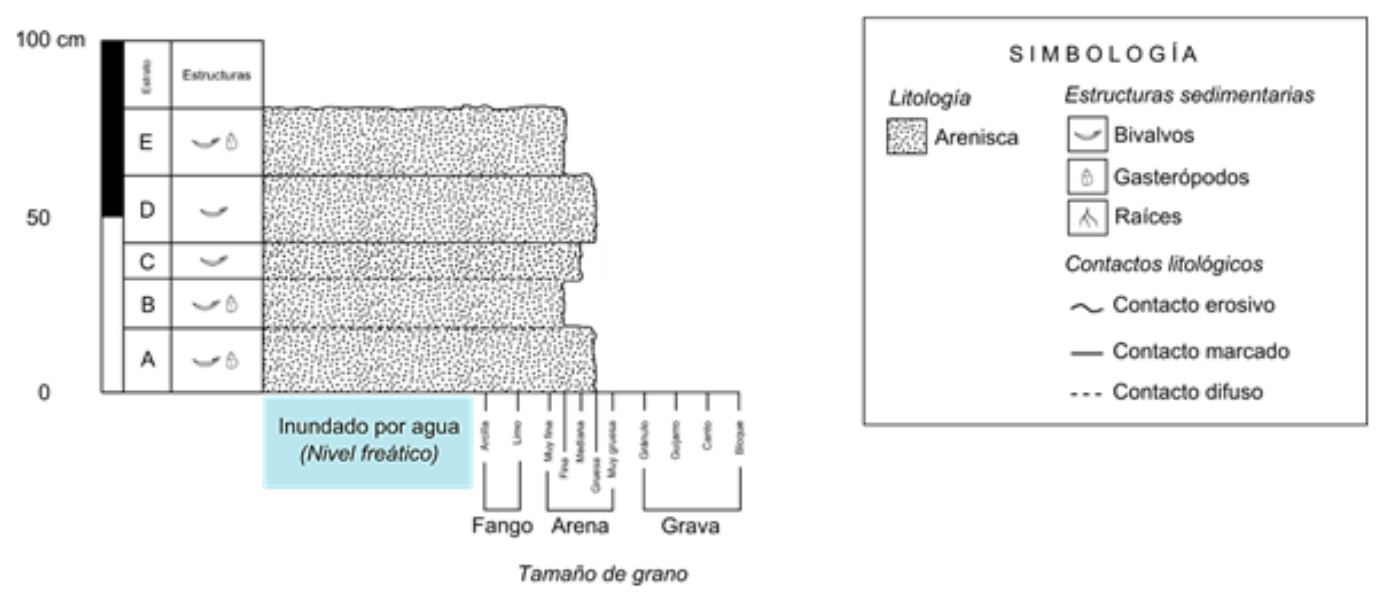

Figura 6. Columna litológica del PV2. 
Estrato D (PV2-D): Dominado por partículas tamaño arenas muy finas a muy gruesas $(3,5 \varphi$ a $2 \mathrm{~mm})$ (Figura 9). Perteneciente al grupo textural Gravelly Sand, clasificado con el nombre del sedimento Fine Gravelly Fine Sand. Composicionalmente dominado por biógenos calcáreos y fragmentos de roca. Presenta un porcentaje de porosidad de $36,5 \%$.
Estrato E (PV2-E): Dominado por partículas tamaño arenas muy finas a finas $(3,5 \varphi$ a $0,25 \mathrm{~mm})$ (Figura 10). Perteneciente al grupo textural Gravelly Sand, clasificado con el nombre del sedimento Fine Gravelly Fine Sand. Composicionalmente dominado por biógenos calcáreos y calcita. Presenta un porcentaje de porosidad de $29,7 \%$.

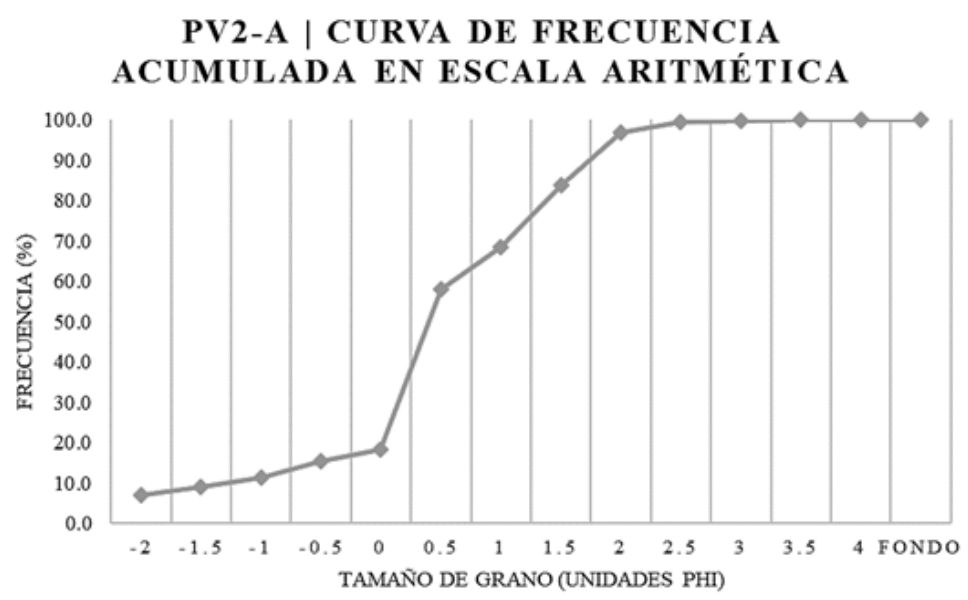

Figura 7. Curva de frecuencia acumulada en escala aritmética del PV2-A.

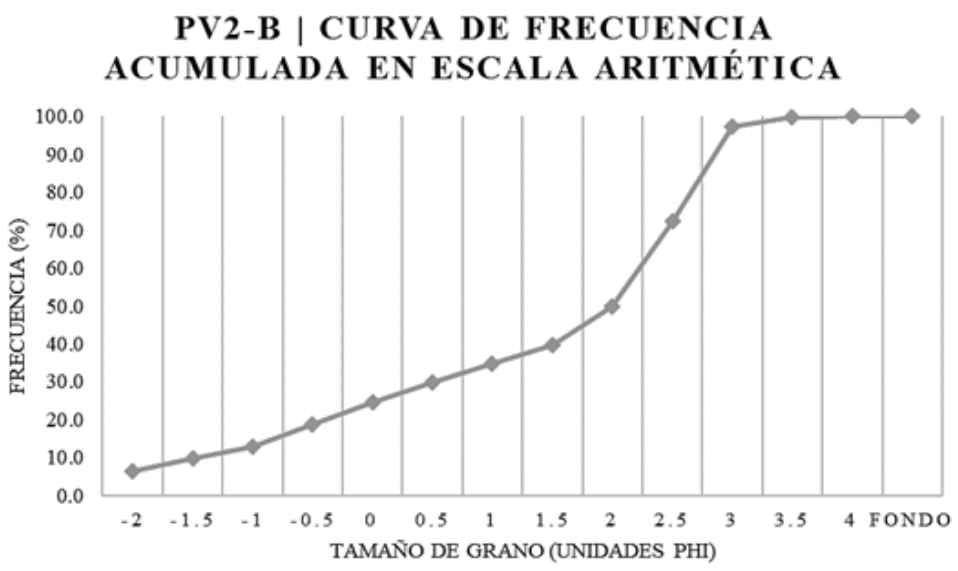

Figura 8. Curva de frecuencia acumulada en escala aritmética del PV2-B.

\subsubsection{PV3 (Perfil vertical 3)}

Se identificó una columna litológica con un espesor de 0,88 m.s.n.m. conformada por 5 estratos. Granulométricamente, domina grava y arenas de $4 \mathrm{~mm}$ a
0,25 mm compuestas por biógenos calcáreos y material detrítico. Se determinó una porosidad con un intervalo de 26,1 a 40,1\% (Figura 11).

Estrato A (PV3-A): Dominado por partículas tama- 


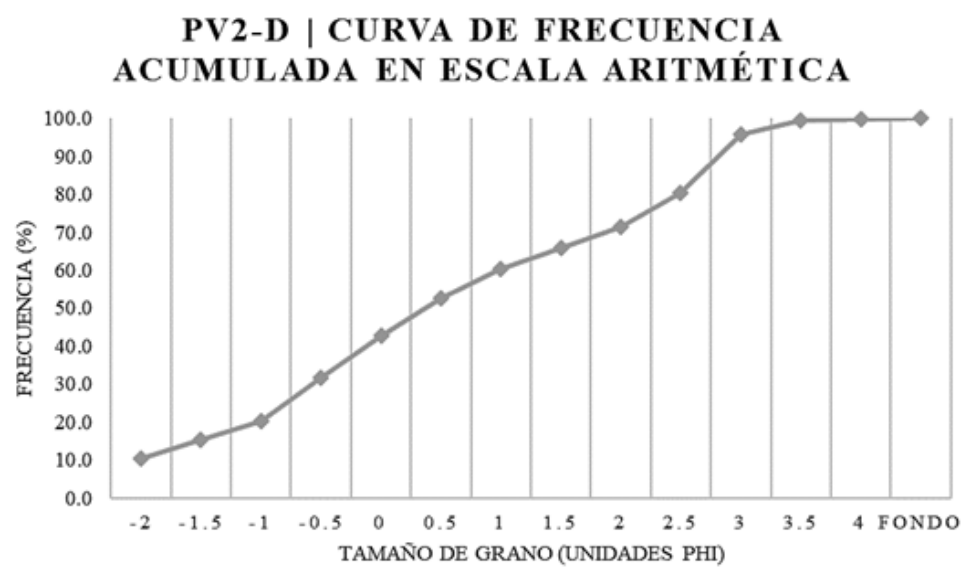

Figura 9. Curva de frecuencia acumulada en escala aritmética del PV2-D.

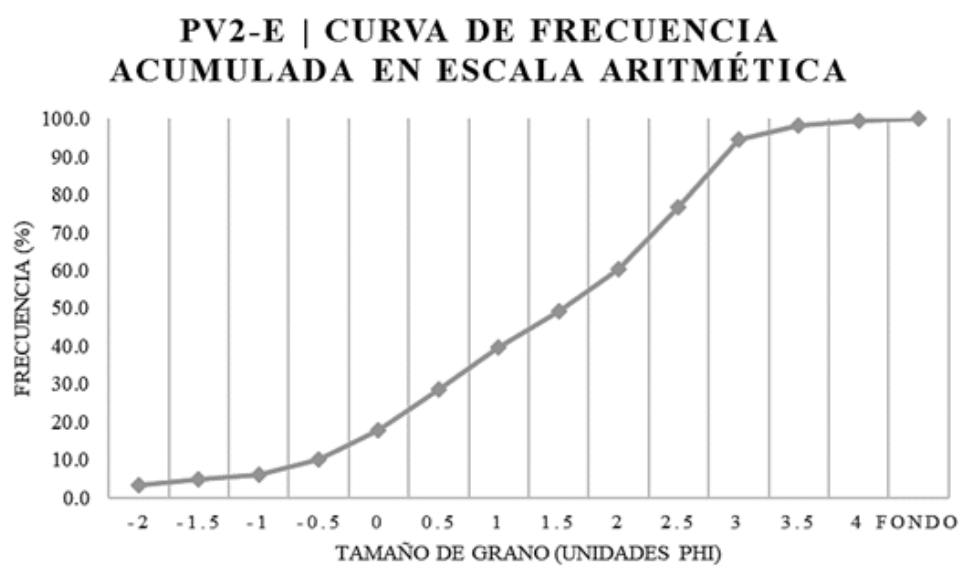

Figura 10. Curva de frecuencia acumulada en escala aritmética del PV2-E.

ño gránulos (2,8 a 4 mm) (Figura 12). Perteneciente al grupo textural Sandy Gravel, clasificado con el nombre del sedimento Sandy Fine Gravel. Composicionalmente dominado por biógenos calcáreos y fragmentos de roca. Presenta un porcentaje de porosidad de $36,8 \%$.

Estrato B (PV3-B): Dominado por partículas tamaño arenas finas a medias $(2,5 \varphi$ a $0,5 \mathrm{~mm}$ ) (Figura 13). Perteneciente al grupo textural Gravelly Sand, clasificado con el nombre del sedimento Fine Gravelly Medium Sand. Composicionalmente dominado por biógenos calcáreos y fragmentos de roca. Presenta un porcentaje de porosidad de $26,1 \%$.

Estrato C (PV3-C): Dominado por partículas tamaño arenas muy gruesas a gránulos $(1,4$ a $4 \mathrm{~mm})$
(Figura 14). Perteneciente al grupo textural Sandy Gravel, clasificado con el nombre del sedimento Sandy Very Fine Gravel. Composicionalmente dominado por biógenos calcáreos y fragmentos de roca. Presenta un porcentaje de porosidad de 33,2\%.

Estrato D (PV3-D): Dominado por partículas tamaño arenas finas a gruesas $(2,5 \varphi$ a $1 \mathrm{~mm})$ (Figura 15). Perteneciente al grupo textural Slightly Gravelly Sand, clasificado con el nombre del sedimento Slightly Very Fine Gravelly Medium Sand. Composicionalmente dominado por biógenos calcáreos y fragmentos de roca. Presenta un porcentaje de porosidad de $36,6 \%$.

Estrato E (PV3-E): Dominado por partículas tamaño arenas medias a gruesas $(1,5 \varphi$ a $1 \mathrm{~mm})$ (Figura 16). 

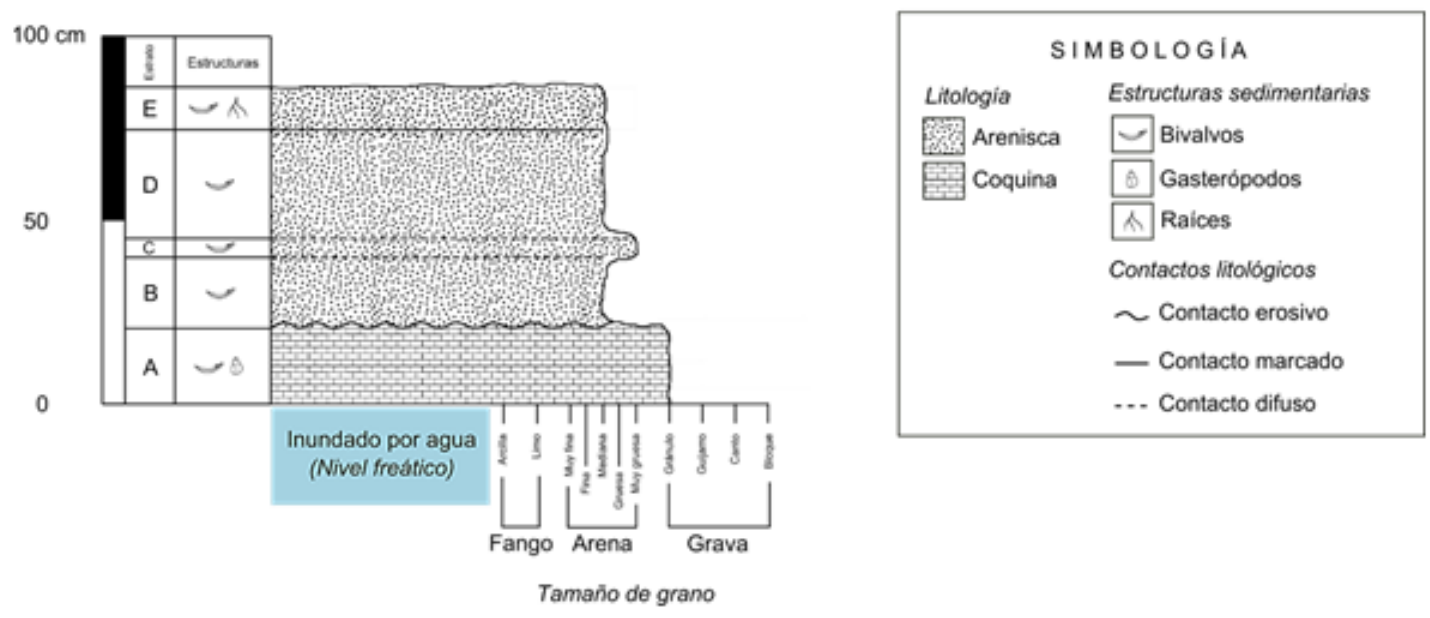

Figura 11. Columna litológica del PV3.

Perteneciente al grupo textural Gravelly Sand, clasificado con el nombre del sedimento Very Fine Gravelly Coarse Sand. Composicionalmente dominado por biógenos calcáreos y materia orgánica. Presenta un porcentaje de porosidad de $40,1 \%$.

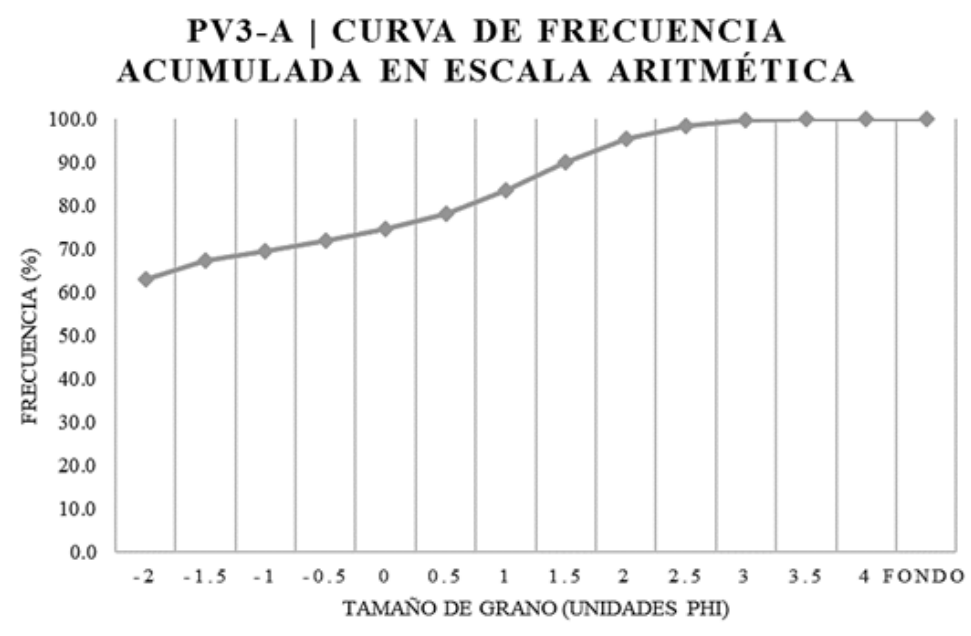

Figura 12. Curva de frecuencia acumulada en escala aritmética del PV3-A.

\subsection{Calidad del agua}

El primer punto de muestreo se localiza a $\pm 481,65 \mathrm{~m}$ del relleno sanitario $\left(18^{\circ} 41^{\prime} 22,452^{\prime \prime} \mathrm{N}, 91^{\circ} 40^{\prime} 45,732^{\prime \prime}\right.$ O), dentro de uno de los cuerpos acuosos de la zona de manglar, lo cual permitió clasificar este punto como una zona de dilución de los lixiviados prove- nientes del relleno sanitario. El análisis de la calidad de agua para este punto de muestreo se describe en la Tabla 1.

Por su parte, el segundo punto de muestreo se localiza a $\pm 1270,46 \mathrm{~m}$ del relleno sanitario $\left(18^{\circ} 41^{\prime} 4,416^{\prime \prime}\right.$ $\mathrm{N}, 91^{\circ} 41^{\prime} 7,62^{\prime \prime} \mathrm{O}$ ), dentro del canal principal (flujo 
laminar con una dirección de corriente N-S) que atraviesa el área de estudio. Las concentraciones de los diferentes parámetros de la calidad del agua para este punto de muestreo se describen en la Tabla 1.

Para el caso de los metales pesados, y considerando los puntos de muestreo como una zona de dilución (MA-Mangla y MA-Canal), se detectaron concentraciones de traza de los metales $\mathrm{Cd}, \mathrm{Cu}, \mathrm{Ni}$ y Zn (Tabla 2).

\section{Discusión}

\subsection{Análisis sedimentológico}

Del análisis granulométrico y componentes sedimentarios de los tres perfiles verticales (PV) se encontró una similitud entre el PV2 y el PV3, ya que ambos presentan un mismo rango de tamaño $(2,5$ $\varphi$ a $2 \mathrm{~mm}$ ) y componentes (biógenos calcáreos y fragmentos de roca), por lo tanto, se determinó una continuidad lateral de los estratos. Por su parte, el primer estrato (PV2-A) del PV2 con el último estrato (PV1-B) del PV1, presentan una semejanza en la granulometría (2,5 phi), componentes sedimentarios (biógenos calcáreos, materia orgánica y cuarzo) y correspondencia en elevación topográfica, lo que permitió correlacionarlos estratigráficamente (Figura 17).

Así pues, los puntos PV1 y PV2 conforman la columna litológica del área de estudio, siendo los PV2 y PV3 la continuación estratigráfica del PV1, y conformando una misma facies sedimentaria arenosa y limosa (Darnell, 2015; Jones, 2015; MagallanesOrdóñez y col., 2015; Ramos-Reyes y col., 2016).

\section{PV3-B | CURVA DE FRECUENCIA ACUMULADA EN ESCALA ARITMÉTICA}

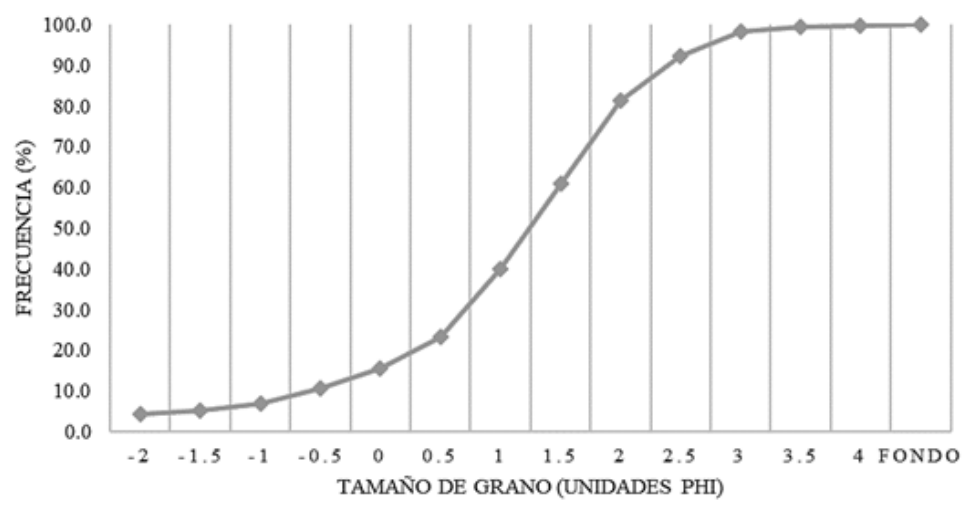

Figura 13. Curva de frecuencia acumulada en escala aritmética del PV3-B.

Por lo anterior, se determinó que el área de estudio está constituida por una columna litológica conformada por seis estratos principales, granulométricamente dominados por arenas de tamaños 0,062 a $4 \mathrm{~mm}$, compuestas por biógenos calcáreos y material terrígeno (Figura 18) Según MagallanesOrdóñez y col. (2015), las arenas podrían provenir del mar; y las arcillas son del continente adyacente.

Los granos en una arena generalmente están en contacto tangencial, formando una red abierta, es decir, tridimensional. Como consecuencia, las are- nas tienen una gran porosidad (cuentan con un sistema de poros lleno de fluido) (Pettijohn, Potter y Siever, 2014). A partir del análisis de las propiedades de masa, se determinó un rango de porosidad $20,2-40,1 \%$, y en función del tamaño de partícula fue posible determinar un rango de permeabilidad de $\pm 10-2-10-4 \mathrm{~m} / \mathrm{s}$. Estos valores permitieron definir que los sedimentos presentan una buena porosidad y una capacidad de permeabilidad muy rápida indicativa de buenos acuíferos, es decir, los fluidos se transportan rápidamente a través del medio poroso (Arellano, 2003; Anovitz y col., 2018). 


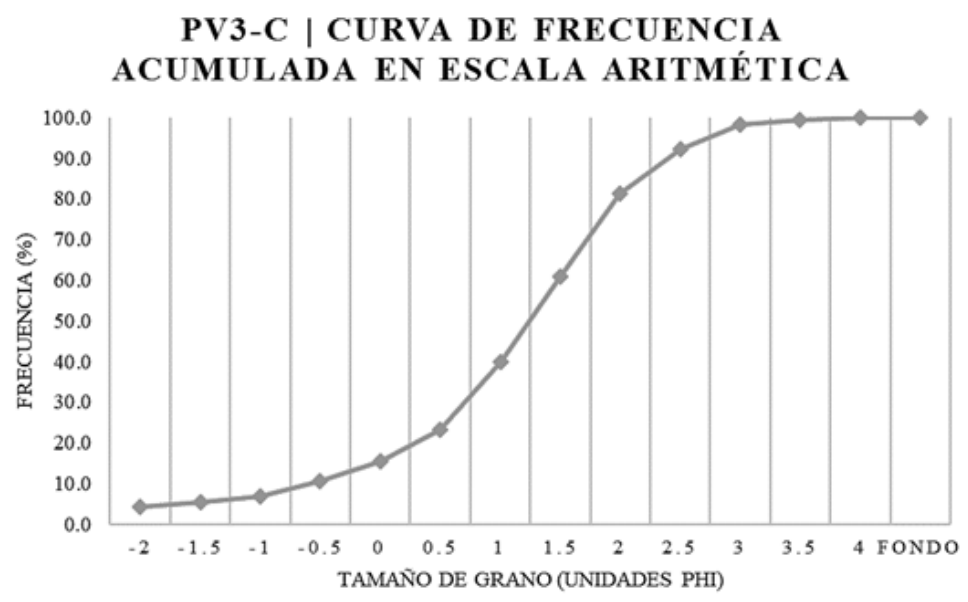

Figura 14. Curva de frecuencia acumulada en escala aritmética del PV3-C.

\section{PV3-D | CURVA DE FRECUENCIA ACUMULADA EN ESCALA ARITMÉTICA}

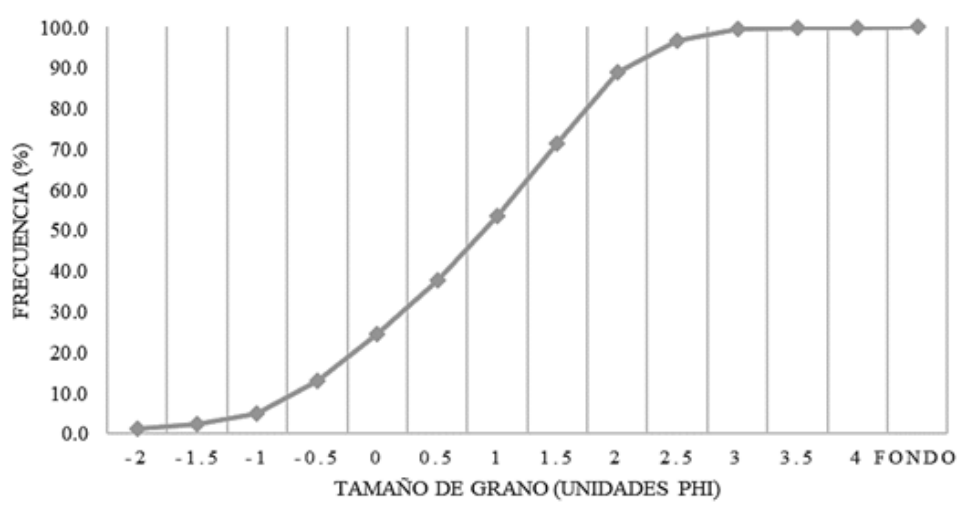

Figura 15. Curva de frecuencia acumulada en escala aritmética del PV3-D.

Tabla 1. Concentraciones de parámetros determinados en la calidad del agua los puntos MA-Manglar y MA-Canal

\begin{tabular}{cccc}
\hline \multirow{2}{*}{ Muestra } & Parámetro & $\begin{array}{c}\text { Concentración } \\
(\mathbf{m g} / \mathbf{L})\end{array}$ & $\begin{array}{c}\text { Límites máximos permisibles } \\
(\mathbf{s e g u ́ n} \text { CONAGUA }(\mathbf{2 0 1 6}))\end{array}$ \\
\hline \multirow{3}{*}{ MA-Manglar } & $D B O_{5}$ & 63,06 & 30 \\
\cline { 2 - 4 } & $D Q O$ & 1338,13 & 40 \\
\cline { 2 - 4 } & Fósforo total & 1,17 & N/A \\
\cline { 2 - 4 } & Nitrógeno total Kjeldahl & 5,49 & N/A \\
\cline { 2 - 4 } MA-Canal & $D B O_{5}$ & $<20$ & 30 \\
\cline { 2 - 4 } & $D Q O$ & 71,94 & 40 \\
\cline { 2 - 4 } & Fósforo total & 1,17 & N/A \\
\cline { 2 - 4 } & Nitrógeno total Kjeldahl & 5,49 & N/A \\
\hline
\end{tabular}




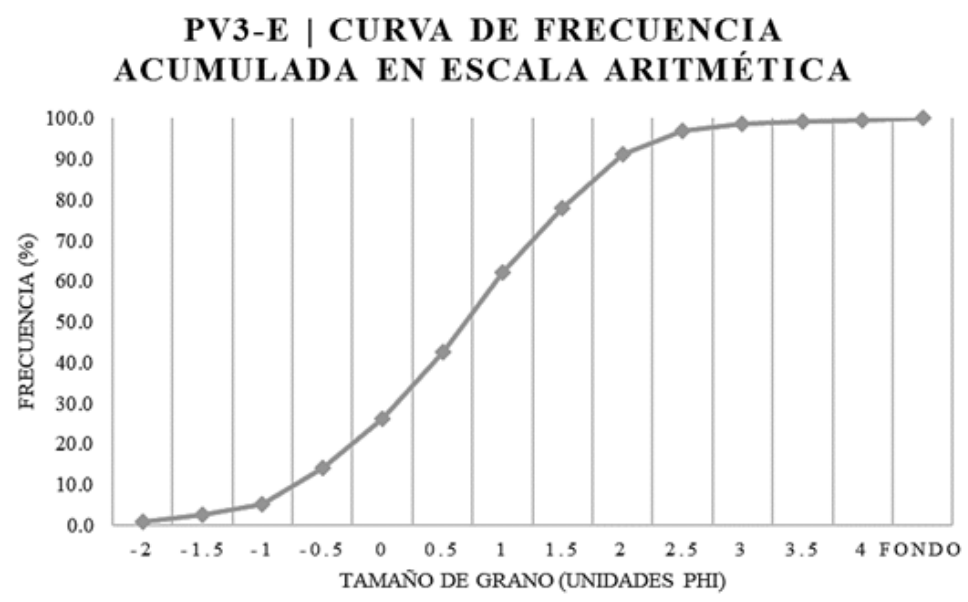

Figura 16. Curva de frecuencia acumulada en escala aritmética del PV3-E.

PERFIL VERTICAL 1 (PV1)

PERFIL VERTICAL 2 (PV2)

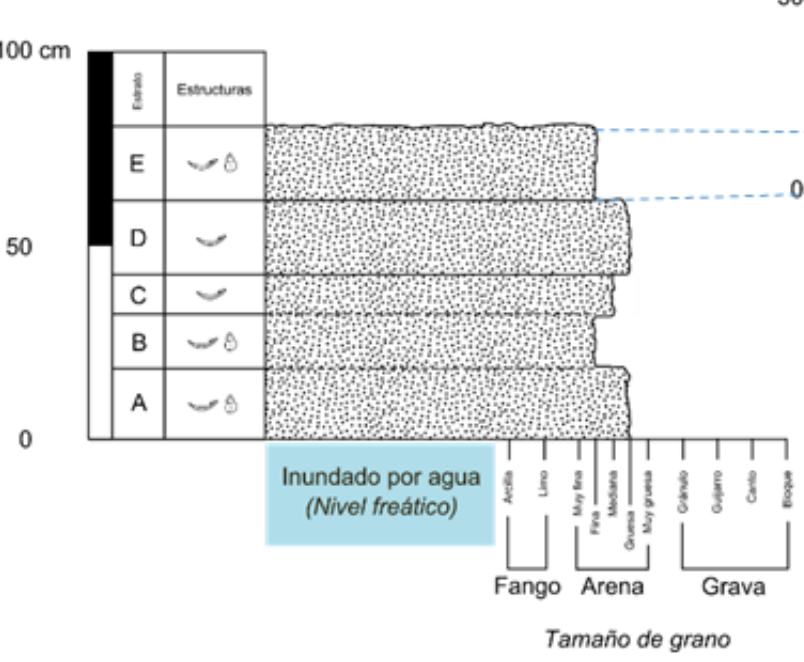

$100 \mathrm{~cm}$

Figura 17. Correlación estratigráfica del PV1-A con el PV2-E.

La dominancia de biógenos calcáreos, así como la evidencia de disolución en algunos granos permitió considerar una porosidad secundaria en los sedimentos. Según Braga, Martín y Puga-Bernabéu (2015), los procesos de disolución incrementan sig- nificativamente la porosidad. Estos procesos incluyen 1) descarga de agua meteórica, 2) disolución alcalina de cuarzo, 3) lixiviación de minerales inestables (por ejemplo, feldespatos, cementos de carbonato y fragmentos de roca) con respecto a fluidos 

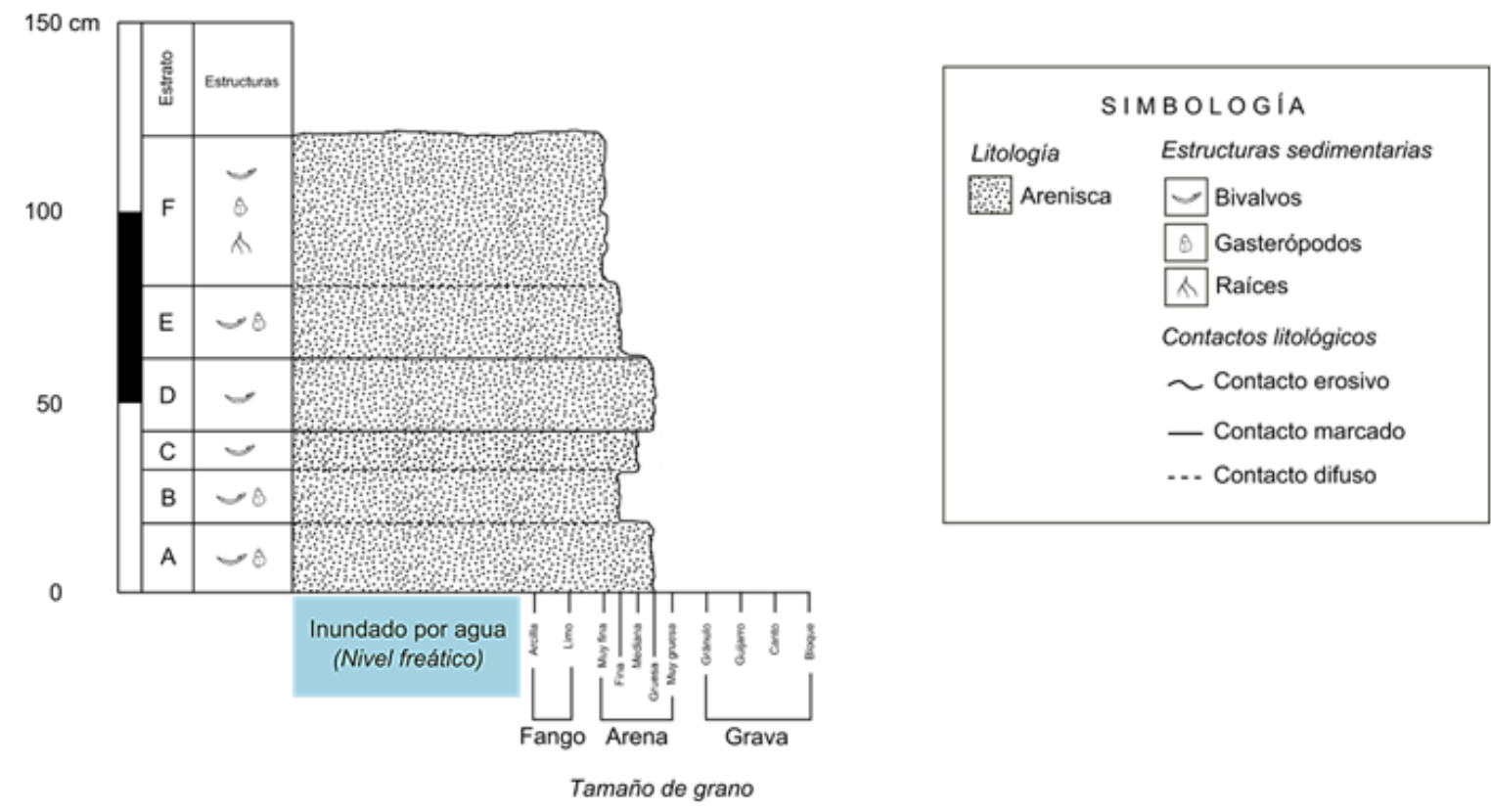

Figura 18. Columna litológica del área de estudio.

ácidos generados por la maduración térmica de la ciones minerales en lutitas adyacentes (Zheng y col., materia orgánica o arcilla cuando se presentan reac- 2015).

Tabla 2. Concentraciones de metales pesados determinados en la calidad del agua de los puntos MA-Manglar y MA-Canal.

\begin{tabular}{cccc}
\hline \multirow{2}{*}{ Muestra } & Parámetro & $\begin{array}{c}\text { Concentración } \\
(\mathbf{m g} / \mathbf{L})\end{array}$ & $\begin{array}{c}\text { Límites máximos permisibles } \\
\text { (según la NOM-001 } \\
\text { SEMARNAT-1996) }\end{array}$ \\
\hline \multirow{3}{*}{ MA-Manglar } & Cadmio & 38 & 0,2 \\
\cline { 2 - 4 } & Cobre & 168 & 6 \\
\cline { 2 - 4 } & Níquel & 0,41 & 4 \\
\cline { 2 - 4 } & Zinc & 3037 & 20 \\
\hline \multirow{3}{*}{ MA-Canal } & Cadmio & 8 & 0,2 \\
\cline { 2 - 4 } & Cobre & 13 & 6 \\
\cline { 2 - 4 } & Níquel & 0,16 & 4 \\
\hline
\end{tabular}

Los flujos de aguas subterráneas juegan un papel importante como medio de transporte para los lixiviados, tanto de forma vertical como de forma horizontal; por tal motivo, son importantes para percibir los riesgos asociados a este flujo (Cortázar, Szantó-Narea y Llamas, 2017; Niño-Carvajal, Ramón-Valencia y Ramón-Valencia, 2016). Por lo anteriormente expuesto, las características sedimentológicas del subsuelo sobre el que se sitúa el relleno sanitario municipal permiten que los lixiviados generados por éste (y acumulados en el medio hidrológico a lo largo de los años), se infiltren y desplacen, direccionándose con un ángulo máximo de $\pm 1,7^{\circ}$ (de manera natural y a partir de la elevación topográfica al punto medio del relleno sanitario) hacia las zonas de escorrentía, es decir, hacia los cuerpos acuosos que conforman la zona de manglar. 
Cabe destacar que uno de los elementos que influencian este transporte es el nivel freático, el cual se encontró a los $\pm 0,87 \mathrm{~m}$ (es decir, clasificándolo como una zona de inundación). Así pues, durante el recorrido vertical y horizontal de los lixiviados, estos se mezclan en el manto freático. Un caso de estudio muy similar a éste es reportado por NiñoCarvajal, Ramón-Valencia y Ramón-Valencia (2016) en el sitio de disposición final de residuos sólidos de El Carrasco, ubicado en el municipio de Bucaramanga, Colombia, en donde se reportó la misma problemática y se encontraron zonas del subsuelo totalmente saturadas por los lixiviados que no fueron manejados de manera adecuada en el sitio de disposición.

\subsection{Calidad del agua}

Considerando los límites máximos permisibles según la NOM-001-SEMARNAT-1996 (SEMARNAT, 1996), y la clasificación para aguas superficiales de la CONAGUA (2016), las concentraciones encontradas correspondientes a los parámetros de DBO5 y DQO son indicativas de cuerpos contaminados y fuertemente contaminados, respectivamente.

Por lo anterior se determinó que la principal fuente de contaminación en los cuerpos acuosos proviene de materiales inorgánicos, siendo el primer punto de muestreo (MA-Manglar) el más representativo debido a la relación de $4,7 \%$ de orgánicos, y $95,3 \%$ de inorgánicos. Por su parte, los valores de Fósforo total y Nitrógeno total Kjeldahl en ambos puntos de muestreo se encuentran dentro de los límites máximos permisibles según la NOM-001SEMARNAT-1996. La concentración de estos parámetros se asocia a la actividad biológica de los manglares que dominan la zona de estudio, ya que tanto el fósforo como el nitrógeno son macronutrientes esenciales en el proceso de fotosíntesis de la biota que conforma el manglar (Bravo-Chaves, PiedraMarín y Piedra-Castro, 2012).

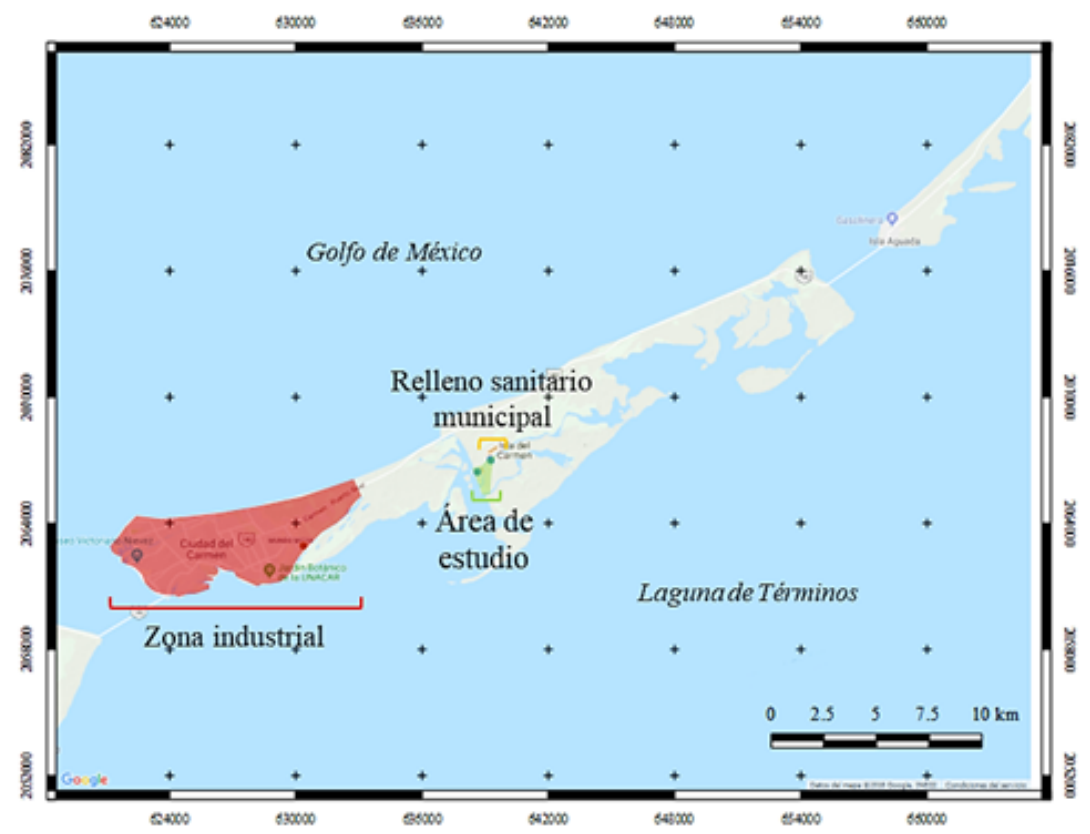

Figura 19. Relación geográfica entre la zona industrial, el relleno sanitario municipal y el área de estudio (Modificado de INEGI (2017).) 
En el caso de los metales pesados, se detectaron concentraciones de trazas de $\mathrm{Cd}, \mathrm{Cu}, \mathrm{Ni}$ y $\mathrm{Zn}$, por debajo de lo establecido en los límites máximos permisibles por la NOM-001-SEMARNAT-1996 (SEMARNAT, 1996). Sin embargo, es preciso destacar que cuando éstos entran en el sistema acuático directamente vía atmosférica o con las aguas de escorrentía, pueden causar impactos graves en concentraciones elevadas debido a su gran toxicidad (Bravo-Chaves, Piedra-Marín y Piedra-Castro, 2012). La disminución evidente en la concentración de metales pesados del punto 2 (MA-Canal) al punto 1 (MA-Manglar) se debe a que el punto 1 se localiza dentro de uno los cuerpos acuosos del manglar, es decir, sin influencia por corrientes marinas internas. Mientras que el punto 2 se ubica en uno de los canales principales del área, en donde las corrientes marinas internas influencian significativamente la disolución de los contaminantes.

El origen de este tipo de contaminación puede ser natural (desgaste de rocas ígneas y meta- mórficas, aerosoles oceánicos y descomposición de detritus) o antropogénico (vertidos industriales y de aguas domésticas) (Bravo-Chaves, Piedra-Marín y Piedra-Castro, 2012). En este caso, la presencia del relleno sanitario, y la ausencia de industrias en la periferia del área de estudio $\left( \pm 127 \mathrm{Km}^{2}\right)$ (INEGI, 2017) (Figura 19), sugiere que el origen de estas concentraciones puede estar asociada a los lixiviados provenientes del relleno sanitario, que infiltran en el subsuelo (altamente poroso y permeable) y se depositan en los cuerpos acuosos de la zona. Los metales pesados son uno de los contaminantes más importantes en ecosistemas acuáticos debido a su toxicidad, persistencia y bioacumulación potenciales. Es bien sabido que los metales pesados poseen un riesgo significativo en la salud humana cuando la dosis de exposición excede los niveles de consumo seguro (Mussali-Galante, Tovar-Sánchez y Castillo, 2013; Zhong y col., 2018). Diversos autores han detectado acumulación de metales tóxicos en tejidos de peces y moluscos de consumo humano (Covarrubias y Peña-Cabriales, 2017).

Tabla 3. Comparativa de los valores de lixiviados y pozo de agua potable de dos rellenos sanitarios típicos con la muestra MAManglar (Modificado de Tavera Cortés (2017), comunicación personal, 06 de diciembre de 2017).

\begin{tabular}{|c|c|c|c|}
\hline Punto de muestreo & Medio & $\mathrm{DBO}_{5}(\mathbf{m g} / \mathbf{L})$ & $D Q O(\mathbf{m g} / \mathbf{L})$ \\
\hline \multirow{2}{*}{$\begin{array}{l}\text { RS “Tultitlán” (Tultitlán, } \\
\text { Edo. de México) }\end{array}$} & Lixiviado 1 & 958562 & 19166667 \\
\hline & Lixiviado 2 & 3717461 & 20833333 \\
\hline $\begin{array}{c}\text { RS "El Milagro" } \\
\text { (Ixtapaluca, Edo. de México) }\end{array}$ & Lixiviado & 1337134 & 45833333 \\
\hline $\begin{array}{c}\text { Pozo de agua potable } \\
\text { (próximo al RS "El Milagro" }\end{array}$ & Agua & $<4,757$ & 2882 \\
\hline $\begin{array}{c}\text { Manglar (MA-Manglar) } \\
\text { (Ciudad del Carmen, Camp.) }\end{array}$ & Agua & 63,06 & 1338,13 \\
\hline
\end{tabular}

A partir de la comparativa de los valores de $\mathrm{DBO}_{5}$ y $\mathrm{DQO}$ identificados en el punto MAManglar (cuerpo acuoso dentro del manglar) con los efluentes de lixiviados de dos rellenos sanitarios municipales y un pozo de agua potable (caso de estudio: Relleno Sanitario "Tultitlán", Tultitlán, Edo. de México y Relleno Sanitario "El Milagro", Ixtapaluca, Edo. de México, M. E. Tavera, comunicación personal, 06 de diciembre de 2017) (Tabla 3), se encontró que tanto la $\mathrm{DBO}_{5}$ como la DQO del punto MA-Manglar se encuentra por debajo de los valores presentados en los lixiviados de un relleno sanitario municipal típico. Por otro lado, para el ca- so del pozo de agua potable próximo a un relleno sanitario municipal, los valores del MA-Manglar se encuentran por encima del mismo, lo cual confirma la determinación anterior al clasificarlo como un cuerpo contaminado según la CONAGUA (2016).

Es importante considerar que la proliferación de algas y macrófitos en función de la carga de nutrientes (nitrógeno $(\mathrm{N})$ y fósforo $(\mathrm{P})$ ) es una de las principales manifestaciones del proceso de eutrofización en los cuerpos acuosos. Los responsables de este fenómeno son principalmente los vertidos de aguas residuales provenientes de actividades antrópicas 
(Ramos, 2018). La eutrofización tiene como efecto adverso un aumento de la biomasa de las algas, la pérdida de oxígeno y la mortandad de algunas especies acuáticas (Espósito y col., 2016).

\section{Conclusiones}

Litológicamente, el área de estudio se encuentra conformada por una facies sedimentaria arenosa y limosa, compuesta por una sucesión de seis estratos de arenas con tamaños de 0,062 a $4 \mathrm{~mm}$, conformados composicionalmente por biógenos calcáreos y material terrígeno. Se determinó un rango de porosidad $20,2-40,1 \%$, y un rango de permeabilidad de $\pm 10-2-10-4 \mathrm{~ms}^{-1}$.

Esta relación, aunada a la disolución de las partículas carbonatadas (dominantes en los sedimentos analizados), permitió determinar que las características sedimentológicas del subsuelo sobre el que se sitúa el relleno sanitario municipal, permiten que los lixiviados generados por éste se infiltren y desplacen a través del mismo, direccionándose (de manera natural y a partir de la elevación topográfica del área mediante un ángulo máximo de $\pm 1,7^{\circ}$ ) hacia los cuerpos acuosos que conforman la zona de manglar. Cabe destacar que uno de los elementos que influencian este transporte es el nivel freático, localizado a $\pm 0,87 \mathrm{~m}$. Así pues, durante el recorrido vertical de los lixiviados, estos se mezclan en el manto freático, lo que representa un impacto negativo al medio ambiente.

Evidencia de lo anterior puede verse reflejado en la calidad del agua, en donde a partir del análisis de los valores de $\mathrm{DBO}_{5}$ y $\mathrm{DQO}$, fue posible clasificar a los cuerpos acuosos (según la CONAGUA (2016) y su comparativa con el monitoreo de los cuerpos acuosos del país) en un rango de "contaminado a fuertemente contaminado", lo cual se atribuye a la elevada concentración de constituyentes inorgánicos (DQO), dentro de los cuales se consideran las concentraciones de traza de metales pesados como cadmio, cobre, níquel y zinc, cuyo origen se relaciona con la presencia del relleno sanitario municipal en el área de estudio.

Por lo anteriormente planteado, existe un impacto ambiental negativo en el área de estudio y se requiere de un plan de manejo integral que mitigue y restaure el ecosistema. Considerando los trabajos de Bravo-Chaves, Piedra-Marín y Piedra-Castro (2012) se propone que la opción más viable sería la clausura del relleno sanitario municipal, y la apertura de uno nuevo que cumpla los lineamientos establecidos en la normativa correspondiente. Sin embargo, Ciudad del Carmen no cuenta con un área que cumpla los requisitos para su selección como relleno sanitario, debido a las características granulométricas del subsuelo y a la somera presencia del nivel freático $( \pm 0,87 \mathrm{~m})$.

\section{Recomendaciones}

1. Caracterizar los residuos asociados a un control de los tipos que se disponen en el relleno sanitario, limitándolos únicamente a residuos sólidos urbanos.

2. Clausurar el relleno sanitario en etapas, planteando una reingeniería en el mismo para implementar nuevas zonas que cuenten con los correspondientes sistemas de impermeabilización y la captación y extracción de lixiviados, considerando el trabajo de Cortázar, Szantó-Narea y Llamas (2017) y NiñoCarvajal, Ramón-Valencia y Ramón-Valencia (2016).

3. Realizar (de forma paralela al punto anterior) el tratamiento y/o valorización de los residuos presentes en el mismo. Es importante destacar que el manejo integral de los RSU, conforme a las normas ambientales, tendrán un aseguramiento en reducir los impactos negativos en el medio ambiente $y$, por ende, en el desarrollo sustentable.

\section{Referencias}

Anovitz, L. M. y col. (2018). «The effects of burial diagenesis on multiscale porosity in the St. Peter Sandstone: An imaging, small-angle, and ultra-small-angle neutron scattering analysis». En: Marine and Petroleum Geology 92, 352-371. Online: https:/ / bit.ly/2yvgjiN.

Arellano, A. Álvarez (2003). Manual de Prácticas del Laboratorio de Sedimentología. Universidad Autónoma de Baja California Sur, págs. 1-83. 
Braga, J. C., J. M. Martín y A. Puga-Bernabéu (2015). Origen de la porosidad y la permeabilidad en sedimentos y rocas carbonatadas. 07/08/2018. URL: https://bit.ly/2A1VqNs.

Bravo-Chaves, F. M. B., G. P. Piedra-Marín y L. P. Piedra-Castro (2012). «Evaluación físicoquímica de los sedimentos en el estero tamarindo y sus tributarios, Guanacaste, Costa Rica». En: Uniciencia 26.1, 41-50. Online: https: / / bit. ly/3gzjwQs.

CONABIO (2012). Sitios de manglar con relevancia biológica y con necesidades de rehabilitación ecológica: Isla del Carmen. 06/06/2018. Comisión Nacional para el Conocimiento y uso de la Biodiversidad. URL: https://bit.ly/2Ao6K6y.

CONAGUA (2016). Monitoreo de Calidad del Agua. 30/03/2018. Comisión Nacional del Agua (CONAGUA). URL: https:/ / bit.ly/2AVza81.

CONANP (2018). Región Planicie Costera y Golfo de México: Área de Protección de Flora y Fauna Laguna de Términos. 14/07/2018. Comisión Nacional de Áreas Naturales Protegidas (CONANP). URL: https:/ / bit.ly/3cffppa.

Cortázar, A. Lobo-García de, M. Szantó-Narea y S. Llamas (2017). «Cierre, sellado y reinserción de antiguos vertederos. experiencias en iberoamérica». En: Revista Internacional de Contaminación Ambiental 32, 123-139. Online: https: / / bit.ly / 2LVpUU3.

Covarrubias, S. y J. Peña-Cabriales (2017). «Contaminación ambiental por metales pesados en México: Problemática y estrategias de fitorremediación». En: Revista Internacional de Contaminación Ambiental 33, 7-21. Online: https: / / bit.ly / 36s8SWS.

Darnell, R. (2015). The American Sea: A Natural History of the Gulf of Mexico. Texas A\&M University Press. 584 págs.

Escudero, M., R. Silva y E. Mendoza (2014). «Beach Erosion Driven by Natural and Human Activity at Isla del Carmen Barrier Island, Mexico». En: Journal Of Coastal Research 71.sp1, 62-74. Online: https://bit.ly/3eB5Vq9.

Espósito, M. E. y col. (2016). «Contaminación natural (As, F) y eutrofización (N, P) en la cuenca del arroyo El Divisorio, Argentina». En: Phyton 85.1, 51-62. Online: https://bit.ly/3d33tbi.

Ghosh, S., D. R. Mishra y A. A. Gitelson (2016). «Long-term monitoring of biophysical characteristics of tidal wetlands in the northern Gulf of Mexico - A methodological approach using
MODIS». En: Remote Sensing of Environment 173, 39-58. Online: https://bit.ly/3bW9G7P.

Honarpour, M. M. (2018). Relative Permeability Of Petroleum Reservoirs. CRC press.

$\mathrm{Hu}$, S. y col. (2017). «Global wetlands: Potential distribution, wetland loss, and status». En: Science of The Total Environment 586, 319-327. Online: https://bit.ly/2XqAWGb.

ICS (2018). International Chronostratigraphic Chart. 02/09/2018. International Commission on Stratigraphy (ICS). URL: https://bit.ly/2yIH2JB.

INEGI (2010). División Municipal. Instituto Nacional de Estadística y Geografía. URL: https: / / bit.ly / 2XFAnbC.

- (2017). Espacio y datos de México. 20/06/2018. Instituto Nacional de Estadística y Geografía. URL: https:/ / bit.ly/3gBpxvM.

Ibáñez-Forés, V., M. D. Bovea y A. Azapagic (2013). "Assessing the sustainability of Best Available Techniques (BAT): methodology and application in the ceramic tiles industry». En: Journal of Cleaner Production 51, 162-176. Online: https: / / bit. ly/3ggHX4V.

Jones, S. J. (2015). Introducing sedimentology. Dunedin Academic Press Ltd. 96 págs.

Magallanes-Ordóñez, V. R. y col. (2015). «Characterization of lithogenic and biogenic zones and natural enrichment of nickel in sediments of the Terminos Lagoon, Campeche, Mexico». En: Estuarine, Coastal and Shelf Science 156, 116-123. Online: https:/ / bit.ly/2LWODsS.

Martínez, A. y col. (2015). «El enfoque de paisajes en la clasificación de humedales. Caso de estudio provincia de Matanzas, Cuba.» En: Verbum 10.10, 79-93. Online: https:/ / bit.ly/36HO7Xr.

Maynard, J. J., R. A. Dahlgren y A. T. O'Geen (2014). "Autochthonous and allochthonous carbon cycling in a eutrophic flow-through wetland». En: Wetlands 34, 285-296. Online: https: / / bit.ly / 2XmIhqe.

Mussali-Galante, P., Ma. Tovar-Sánchez E.and Valverde y E. Rojas del Castillo (2013). «Biomarcadores de exposición para determinar la contaminación ambiental por metales pesados: de las moléculas a los ecosistemas». En: Revista internacional de contaminación ambiental 29.1, 117-140. Online: https:/ / bit.ly /2XdyPXb.

Nahlik, A. y S. Fennessy (2016). «Carbon storage in US wetlands». En: Nature Communications 7, 1-9. Online: https:/ / go.nature.com/2LRFArs. 
Niño-Carvajal, L. X., J. A. Ramón-Valencia y J. L. Ramón-Valencia (2016). «Contaminación fisicoquímica de acuíferos por los lixiviados generados del relleno sanitario El Carrasco, de Bucaramanga». En: Producción + Limpia 11.1, 66-74. Online: https://bit.ly/2M8nYHR.

Palacio-Prieto, J. L., M. A. Ortíz-Pérez y A. GarridoPérez (1999). «Cambios morfológicos costeros en Isla del Carmen, Campeche, por el paso del huracán Roxanne». En: Investigaciones Geográficas (Mx) 40, 48-57. Online:https:/ / bit.ly/2ZKrSyT.

Pettijohn, F. J., P. E. Potter y R. Siever (2014). Sand and sandstone. New York: Springer Science \& Business Media.

Rains, M. C. y col. (2016). «Geographically isolated wetlands are part of the hydrological landscape». En: Hydrological Processes 30, 153-160. Online: https://bit.ly/3cfFnsX.

Ramos-Reyes, R. y col. (2016). "Indicadores geomorfológicos para evaluar la vulnerabilidad por inundación ante el ascenso del nivel del mar debido al cambio climático en la costa de Tabasco y Campeche, México». En: Boletín de la Sociedad Geológica Mexicana 68.3, 581-598. Online: https: //bit.ly/2Aq48Fh.

Ramos, A. (2018). «Evaluación del riesgo de eutrofización del embalse El Quimbo, Huila (Colombia)». En: Revista Logos Ciencia y Tecnología 10.2, 172-192. Online: https://bit.ly/3gK8oQW.

Rendón-Macías, M. E., M. A. Villasís-Keeve y M. G. Miranda-Novales (2016). «Estadística descriptiva». En: Revista Alergia México 63.4, 397-407. Online:https:/ /bit.ly/36OMTda.

SCFI (1980a). Norma Mexicana NMX-AA-003-1980. Aguas residuales. - Muestreo. Inf. téc. 25 de marzo de 1980. Secretaría de Comercio y Fomento Industrial. Diario Oficial de la Federación, Online: https://bit.ly/3ch4vzs.

- (1980b). Norma Mexicana NMX-AA-014-1980. Cuerpos receptores. - Muestreo. Inf. téc. 5 de septiembre de 1980. Secretaría de Comercio y Fomento Industrial. Diario Oficial de la Federación, Online: https://bit.ly/36GjGB7.

- (2001a). Norma Mexicana NMX-AA-028-SCFI2001. Análisis de agua - Determinación de la Demanda Bioquímica de Oxígeno en aguas naturales, residuales (DBO5) y residuales tratadas - Método de prueba (cancela a la NMX-AA-028-1981). Inf. téc. Secretaría de Comercio y Fomento Industrial. Diario Oficial de la Federación, Online: https: //bit.ly/2zHuoet.
- (2001b). Norma Mexicana NMX-AA-029-SCFI2001. Análisis de aguas - Determinación de Fósforo total en aguas naturales, residuales y residuales tratadas - Método de prueba (cancela a la NMXAA-029-1981). Inf. téc. Secretaría de Comercio y Fomento Industrial. Diario Oficial de la Federación, Online: https:/ / bit.ly/2XgaQH3.

- (2011a). Norma Mexicana NMX-AA-026-SCFI2010. Análisis de agua - Medición de Nitrógeno total Kjeldahl en aguas naturales, residuales y residuales tratadas - Método de prueba - (cancela a la NMXAA-026-SCFI-2001). Inf. téc. 3 de marzo de 2011. Secretaría de Comercio y Fomento Industrial. Diario Oficial de la Federación, Online: https: / / bit.ly/2XW3YOn.

- (2011b). Norma Mexicana NMX-AA-030/2-SCFI2011. Análisis de agua - Determinación de la Demanda Química de Oxígeno en aguas naturales, residuales y residuales tratadas - Método de prueba - Parte 2 -determinación del índice de la Demanda Química de Oxígeno - Método de tubo sellado a pequeña escala. Inf. téc. 27 de junio 2013. Secretaría de Comercio y Fomento Industrial. Diario Oficial de la Federación.

- (2016). Norma Mexicana NMX-AA-051-SCFI2016. Análisis de agua. - Medición de metales por absorción atómica en aguas naturales, potables, residuales y residuales tratadas- Método de prueba (cancela a la NMX-AA-051-SCFI-2001). Inf. téc. 7 de diciembre de 2016. Secretaría de Comercio y Fomento Industrial. Diario Oficial de la Federación, Online: https:/ / bit.ly/3euPT0F.

SEMARNAT (1996). Norma Oficial Mexicana NOM001-SEMARNAT-1996. Que establece los límites máximos permisibles de contaminantes en las descargas de aguas residuales en aguas y bienes nacionales. Inf. téc. 6 de enero de 1997. Diario Oficial de la Federación. Secretaría de Medio Ambiente y Recursos Naturales, Online: https: / / bit.ly / 2TSWzhl.

- (2000). Norma Oficial Mexicana NOM-021SEMARNAT-2000. Que establece las especificaciones de fertilidad, salinidad y clasificación de suelos. Estudios, muestreo y análisis. Inf. téc. 31 de diciembre de 2002. Secretaría de Medio Ambiente y Recursos Naturales. Diario Oficial de la Federación, Online: https: / / bit.ly/3gKd6y6.

- (2003). Norma Oficial Mexicana NOM-083SEMARNAT-2003. Especificaciones de protección ambiental para la selección del sitio, diseño, construcción, operación, monitoreo, clausura y obras comple- 
mentarias de un sitio de disposición final de residuos sólidos urbanos y de manejo especial. Inf. téc. 04 de agosto de 2015. Secretaría de Medio Ambiente y Recursos Naturales. Diario Oficial de la Federación, Online: https:/ / bit.ly/2XeZSSg.

- (2009). Manual de especificaciones técnicas para la construcción de rellenos sanitarios para residuos sólidos urbanos (RSU) y residuos de manejo especial (RME). Inf. téc. 22/05/2018. Secretaría de Medio Ambiente y Recursos Naturales, Online: https: / / bit.ly/2TRSUjP.

SGM (2005). Carta geológico-minera, Ciudad del Carmen E15-6, Campeche y Tabasco. 15/04/2018. Servicio Geológico Mexicano (SGM). URL: https: / / bit.ly/2ZPQqWU.
Tavera Cortés, M. E. (2017). Gestión Ambiental y Residuos Sólidos Urbanos. México CDMX: Colofón.

Zheng, J. y col. (2015). «Relationships between permeability, porosity and effective stress for lowpermeability sedimentary rock». En: International Journal of Rock Mechanics and Mining Sciences (IJRMMS) 78, 304-318. Online: https: / / bit.ly / 2LWCA1F.

Zhong, W. y col. (2018). «Health risk assessment of heavy metals in freshwater fish in the central and eastern North China». En: Ecotoxicology and Environmental Safety (EES) 157, 343-349. Online: https://bit.ly/2LYNG1i. 